\title{
Anatomical Data Analysis and Normal Femur Bone Reconstruction for Designing Titanium Alloys Total Hip Joint Femoral Stem
}

\author{
Nandang Suhendra*, Dwi Gustiono, Masmui, Gina Nastia \\ Center for Materials Technology - BPPT, Puspitek - Serpong 15314, Indonesia \\ *Corresponding Author: nandang_suhendra@bppt.go.id
}

Article history :

Received 16 November 2015

Accepted 01 December 2015

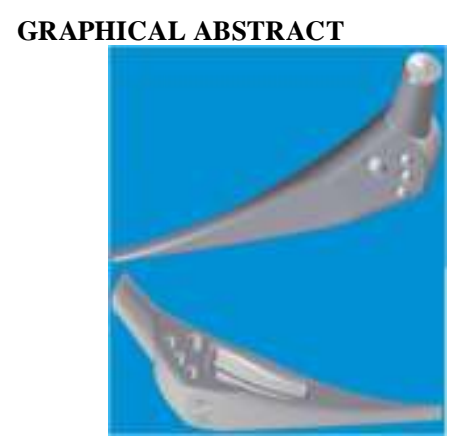

\begin{abstract}
The purpose of the work is to obtain size and geometry numerical data of femur bone for designing femoral stem of total hip joint arthroplasy to fit for Indonesian patient with normal hip joint structure. Geometry data of femur bone was taken by both X-ray and CT scan methods. The X-Ray anatomy digital objects were taken from different angles at certain intervals as input data to reconstruct anatomical objects. Slicing of femur bone imaging taken by CT scan has a constant interval. Data obtained from both CT scan and X-ray were compared. A solid model of bone is used as a visual-tactile representation, planning surgery (surgical) and simulation tool for physicians. In this work the data input was used for design of a total hip joint arthroplasty (THA) femoral stem. The work reported in this article is considered as a preliminary stage of the development of total hip joint arthroplasty devices for being fit to local (Indonesian) patients with hip joint disorders. The preliminary designs developed in this work is suit for many kind materials, however, for the implementation into manufacturing works, the use of Titanium Alloys need to consider for appropriate femoral stem designs by taking into account the available of tools and effectiveness of femoral stem production, made of the materials.
\end{abstract}

Keywords: CT-scan, X-Rays, hip joint, femur reconstruction, Titanium Alloys femoral stem

(C) 2015 Penerbit UTM Press. All rights reserved http://dx.doi.org/10.11113/mjfas.v11n4.392

\section{INTRODUCTION}

The need of a proper implant-patients match in total hip-joint arthroplasty, especially, for cementless femoral stems has been reported to be a crucial thing that orthopedic surgeons concern about. Some of the complications of mismatch are aseptic loosening, improper load distribution, and discomfort. In this work, design and manufacture of total hip joint arthroplasty femoral stem is of our target to be completed. With taking a consideration of size and geometry mismatch between implant and femur bone problem faced by Indonesian and global orthopedists, the early stage of this work is to analyze dimension (size and geometry) of patient anatomy, especially hip joint part.

Several researchers have reported their works with purpose for the reconstruction of human bone anatomy. Both CT Scan and X-Ray image data have been used to obtain geometrical data of femur. The X-Ray anatomy digital objects could be taken from different angles at certain intervals as input data to reconstruct anatomical objects. Slicing of femur bone imaging taken by CT scan has a constant interval. Data obtained from both CT scan and X-ray were compared using slices taken with a constant distance between the slices. A solid model of bone is used as a visual-tactile representation, planning surgery (surgical) and simulation tool for physicians. In this work this technique was used as data input for the design of a femoral stem of a total hip joint arthroplasty (THA).

Rawal, et, al. [1] described a methodology for implant designing from measured anthropometric data, fit evaluation, finite element (FE) stress analysis and subsequently implant manufacturing using the CAM (Computer Aided Manufacturing) technique. They analyzed differences in dimensions between femurs of elderly Indians and those of populations from other regions; and then compared them with purposes to seek a way to solve the problem of a possible geometric mismatch between a selected implant and the femurs of Indian patients as of surgeons presently concerned.

Over sizing in component of total hip-joint implant could give risk especially prevalent among East Asian and other Asian subpopulations. It is known that population in those region, including Indonesia have smaller build and stature as compared with their Middle East and Western counterparts, as the prostheses have been typically designed based on western anthropometric data[2, $3]$. 
It is also essential to optimize physiological load transfer and techanical stability, a close geometric fit between cementless stems and the bone stock $[4,5]$. To achieve better fit of the stem in the proximal femur, it is more desirable to go for a customized implant for individual femora[6, 7].

In implantation of a total hip joint arthroplasty, destruction of the topmost femur section can occur, and this manifests itself in an extremely thin or even partially absent cortical substance [8]. If, in such a state, a reoperation has to be carried out, this section cannot be used for supporting the new prosthesis. For this case, the state of the art makes two prosthesis versions available. One of these versions is based on the replacement of the degenerated, upper section of the femur, which is removed and replaced by bone prosthesis [9]. The latter is supported on the resection plane and its stem is anchored in the distal, remaining part of the bone. The disadvantage of this version is the loss of the natural muscular attachment in the trochanter region and the restriction of further prosthetic measures in the future. Not infrequently, this causes total loss of the femur bone $[10,11]$.

The second, more frequently used version comprises bone prosthesis with a long stem, which reaches down beyond the degenerated section of the femur bone and is cemented in deeply in the distal section of the bone. In this case, the disadvantage is that, as a rule, secure support for the neck of the prosthesis resting on the bone in the weakened section of the femur section cannot be achieved [12]. Thus, there is a risk of the prosthesis subsiding into the femur bone. Due to the absence of support of the upper prosthesis part, the latter is subjected to a higher oscillating load and tends to an increased risk of fracture. It has been proved that, for biomechanical and biological reasons, total filling of the degenerated bone section with cement provokes complete failure of the residual remaining, thin cortical substance [13].

Regeneration of the weakened bone section can be achieved only by a cement-free implantation technique, with condition of there is no movement between the prosthesis and the bone, and this presupposes stable anchorage of the prosthesis in the lower bone section, with prevention of subsidence of the prosthesis [14]. It was reported that regeneration of weakened bone section could be achieved when the transverse surface, supported on the bone, of the prosthesis is formed by a plurality of support parts engaging in the bone at mutual spacing ${ }^{[15]}$. The interspace formed between the supporting parts makes it possible for a bone connection to remain or to be reformed between the femur bone sections located below and above the supporting parts [15].

The support of the prosthesis, apart from the supporting components in the bone at mutual spacing, an annular support surface can also be used. The external diameter of prosthesis supporter is smaller than that of the bone at the particular point [11]. In many situations the support parts engaging in the bone at mutual spacing merely could sufficiently lie in one plane; even thought, the use of support parts in several planes is also possible.

The use of ring in implantation of femoral stem is after the femur bone has been completely installed. The prosthesis stem is then knocked into the medullary canal. The ring is thus positioned within the medullary space, so that linear growth of the cortical bone starting from the distal side towards the end of proximal side is possible.

The lower section of femoral stem prosthesis is introduced by putting it into femur bone. The upper part of the stem has a slightly greater diameter than that of the lower, so that a tapered contact surface is formed at the transition point by a change in diameter. The lower section of the stem has longitudinal grooves. The purpose of the longitudinal groves formation is made to achieve a greaterarea of interface between femoral bone and implant materials.

The upper section of the stem is designed to be releasable joint (femoral stem with modular type design). Femoral neck connects femoral head with femoral stem parts [16]. The joint is accomplished as a result of the neck part having a sleeve which encloses and fits around the upper stem for improved transmission of the bending moments. The mutually opposite end faces of the prosthesis stem and of the sleeve bottom have mutually engaging, radial teeth which allow a rotationally secure joint by means of the tension screw in various angular positions, in order to adjust the desired anteversion. The tension screw can be secured against undesired twisting by means of a known locking device in a bore. Adaptors allow a desired spacing between the joint head and the contact surface to be set.

In the implanted state, the prosthesis is supported via the contact surface on a support ring which has a bore fitting the contact surface and from which several support parts or ribs radiate as circumferentially spaced apart radial projections. The external diameter of the support ring carrying the support parts is greater than of the internal diameter of the medullary canal, but smaller than that of the external diameter of the femur bone. Therefore, both the support ring and the projections can be supported on the resection surface of the femur bone. At the same time, this leaves sufficient interspace in the form of a plurality of longitudinally extending channels or paths between the support projections in the cross-sectional region of the bone, through which interspace the bone material can connect the upper section and the lower section of the femur bone. Spacer rings or shims are provided for adjusting the distance between the contact surface 5 and the support ring [17].

The operation procedure is such that, at a height at which the cortical substance of the femur bone is of sufficient thickness, the femur bone is severed. The supporting ring is interposed at this point. The prosthesis stem is then knocked into the medullary canal from the proximal end ${ }^{[17]}$. The upper, thinned femur section is filled with natural, homologous or autologous bone material, and 
the neck part is put in place on the prosthesis stem and fixed in the desired anteversion position. The adaptors and/or then allow correct length adjustment. It can be provided that the ring part of the support ring is located predominantly or exclusively within the medullary canal, so that the greatest possible cross-sectional area is left between the support projections for the cortical substance to grow through longitudinally [8].

The patient's leg is strapped into a traction table that gives distraction of the hip joint, pulling the joint open in order to allow the passage of the arthroscope (a small rigid telescope with a digital camera on the end) into the space between the ball (femoral head) and socket (acetabulum) of the joint. Small probes and other specialised instruments that can be introduced into the hip joint to allow a wide variety of different surgical procedures to be performed.

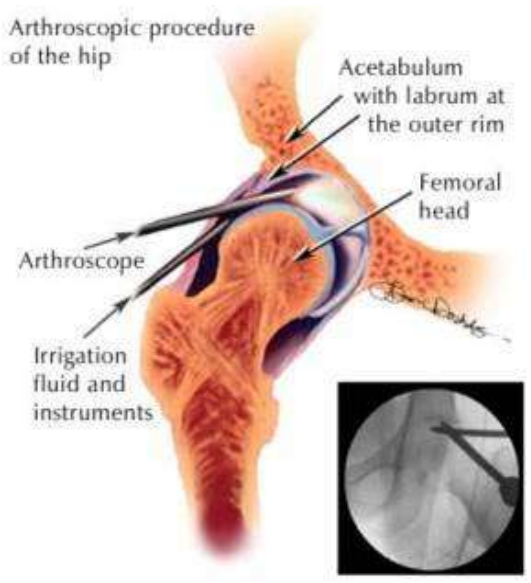

Figure.1 Diagram showing how a telescope is inserted into the hip joint (inset picture shows an X-ray view of the telescope and a probe in the hip joint) [18]

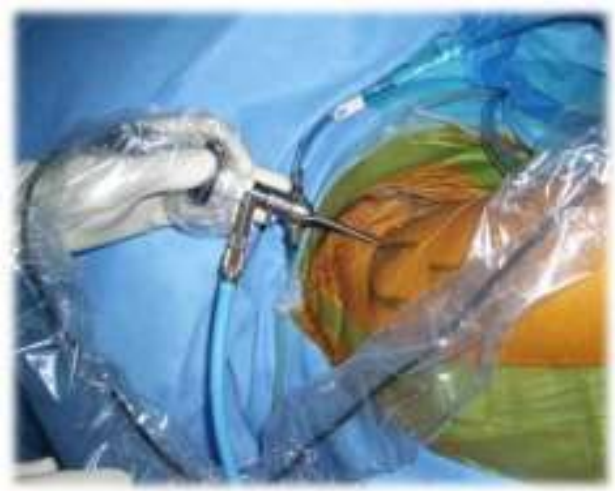

Figure.2 Intra-operative picture showing a hip arthroscope inserted into a hip joint [18]

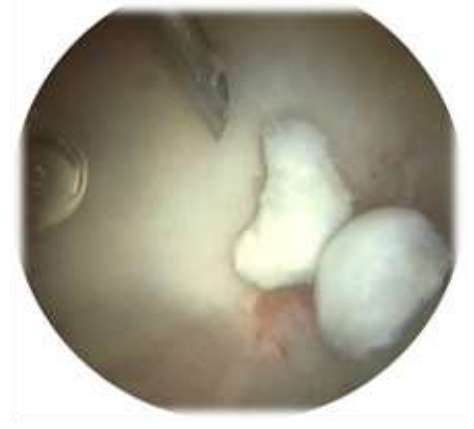

Figure.3 Intra-operative arthroscopy picture showing two loose pieces of cartilage within a hip joint just before they are surgically removed [18].

The primary objectives of this paper are: 1) To collect femur bone in the region of normal hip joint by the use of X-Ray; 2) To observe femur bone which is taken from upper knee to hip joint area, with a particular slice thickness; 3) To reconstruct femur bone from the CTimaging; 4) To design femoral stem and modullary canal of the femur bone; 5) To computerized designing and modeling total hip joint replacement.

\section{EXPERIMENTS}

\subsection{X-Ray Observation}

Experiments were performed to study the shape of femoral bone by Taking X-Ray images/ pictures from normal human femur bone. The geometry of collected pictures then measured and analyzed for further investigation and for femoral bone reconstructions.

As a result of the bone reconstruction achievable according to the reported works, measures and reoperations which may become necessary later are facilitated considerably and the risk of total loss of the femur bone is substantially reduced [6].

\subsection{CT-Scan Imaging}

The femoral bone of normal patients were scanned using CT-scan technique. Computed Tomography scan (CT scan, previously known as a CAT scan) is a scan that uses $\mathrm{x}$-rays and a computer to create images of the body in great detail. In hospitals it is used to image all sorts of medical and surgical problems, but for sports medicine, it is mostly used to look at bones that have been affected by arthritis, injury or surgery. It cannot distinguish soft tissues in as much detail as MRI or ultrasound but it is better for looking at certain conditions of bone (including fractures), and for finding loose material within joints[18] 


\subsection{Femur Bone Reconstruction}

The maximum stem-cortical bone contact plays a dominant role in the long-term fixation of the prosthesis; narrowing stem-cortical bone space and the bony ingrowths reduces the reaction to debris due to wear, the particle flows caused by the wear debris, the bone resorption, and the growth of pseudo-membrane. It was realized that the endosteal contour varies significantly from person to person. Thus, only one common designed prosthesis is not sufficient to achieve a high degree of accuracy in terms of modeling the proximal femur, therefore computer-assisted reconstruction of a three-dimensional canal model of the femur is a very promising way to produce custom hip stem prostheses.

A specially developed image-processing program recognized the edge of each $\mathrm{CT}$ image according to the different contrast between the edges and other parts shown on the image. The subsequent edge image processing and graphics were performed using coordinate on $\mathrm{X}$-Y axes based on the gauge of the CT image obtained. This edge detection method picked out the natural edge of the canal, examined the obtained images, refined the contour of the canal and eventually represented the periosteal and endosteal contours of the femur of each CT slice[2, 18]. These data were saved for later three-dimensional construction. The analysis of the canal contour in the upper part of lesser trochanter needed to be done by both automatic edge detection program and manual edge detection by the designer due to the complex contour of canal in the region above the lesser trochanter where the calar exists[1, 2, 6].

The endosteal contour data of the proximal femur were input into an automatic computerized contouring program (Solidworks software). This program generated the three-dimensional coordinates composed of X-, Y-, and $\mathrm{Z}$-axes based on the gauge of CT image. By choosing 36 points on each obtained endosteal contour, and then connecting them into naturally looking contours, new endosteal contours were obtained on these coordinates. The distance between the adjacent planar contours was $3.0 \mathrm{~mm}$. The reconstruction of the three-dimensional contours generated an accurate model of the proximal femoral canal. According to the obtained model, the stem-design software generated a prosthesis stem shape that was exactly the same as the reconstructed canal model. The distal part of the stem was pulled through the obtained canal model from the proximal end of the model along the vertical axis. The overlapping stem regions were removed by redefining them at the intersections with the canal. This placed the stem once again completely within the canal, and the stem was elevated to the next level and the process repeated until the entire stem had passed through the proximal neck cut. The resultant stem shape described the optimal stem canal fit that was still insertable. The reconstructed threedimensional stem-canal model can be displayed and viewed in any plane, at any level. After this step, a prosthesis neck needed to be designed in the proximal stem part and the distal part of stem needed to be modified into a round shape, at this point, a complete femur prosthesis (not including the head) came into being.

\section{RESULTS AND DISCUSSION}

\subsection{X-Ray Images}

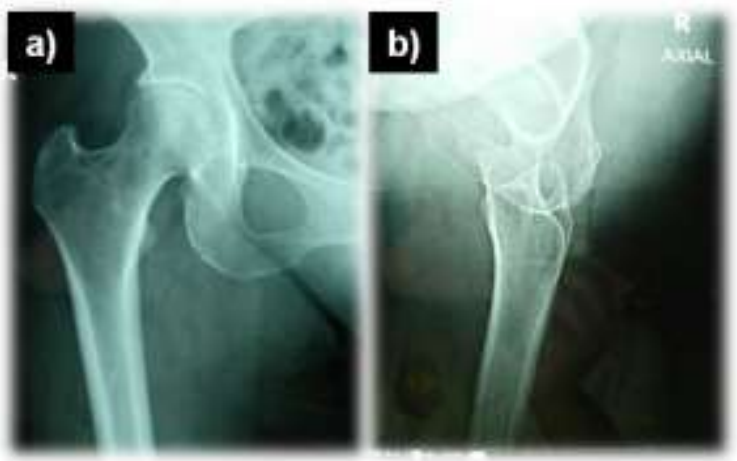

Figure.5 X-Ray Imaging of a normal Femoral bone

Typical X-ray imaging of the original femoral bone prior to manipulate is shown in Figure 5. It can be seen from Figure 1 that on the right side of a normal femoral bone well fitted to the pelvic bone. Figure 5 was taken from a RSU Dr. Soetomo General Hospital - Surabaya with normal femoral bone.

\subsection{CT- Images of Femoral Bone}

CT-scan observations of the femur bone. Bone is seen as bright white and is seen in great internal detail. Although muscles and tendons can be seen, this is not the best scan for these. CT is best for looking at bone fractures, and a fracture can be seen on this picture (Figure 6).

\subsection{Femur Reconstruction}

Further work was to reconstruct femur bone. The cross-sectional shapes of the canal below the trochanter were round or elliptic. The contrast between the cortex and canal showed on CT were clear, therefore it was easy for a computer to perform the edge-detection and obtain a continuous contour. The rendered three-dimensional canal model had a lateral " $\mathrm{s}$ " shaped curvature, and the upper part was wider than the lower part, which was shaped like a funnel. After the extraction process, a contour of the prosthesis was obtained. In the insertion process, the contour of the stem appeared similar to the canal, but the former was only optimally within the latter's contour.

Solid-works program could use the data that were obtained by the method above discussed in designing the prosthesis. The computer-assisted reconstruction of the three-dimensional canal model represented the original shape of the stem canal. After modifications to the distal 
and proximal parts of the original stem model, the prosthesis could be applied in clinical applications.
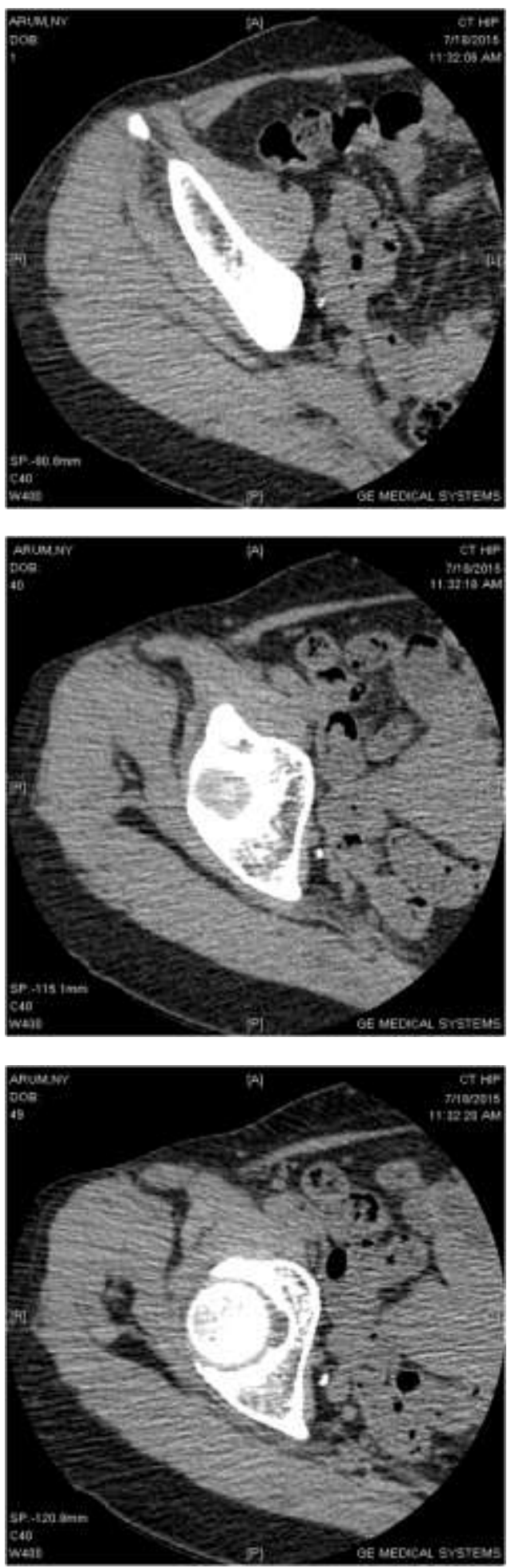

Figure. 6 Computerized Tomography Scan of femur bone
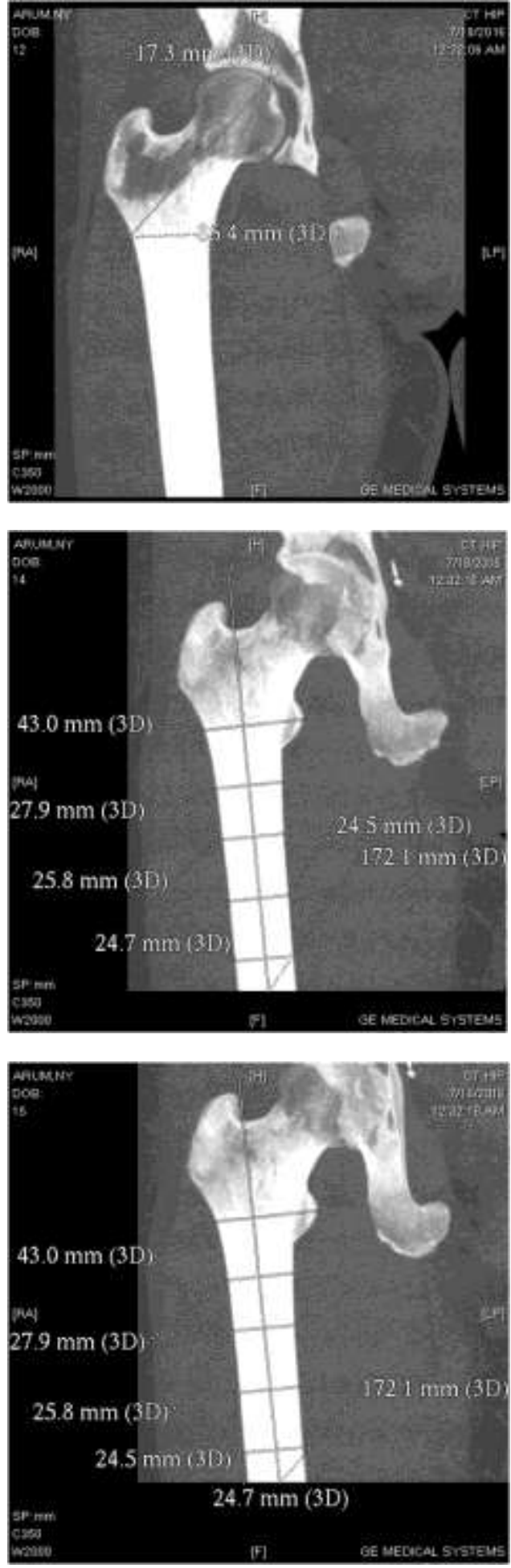

Figure.7 Reconstruction of femur bone 
Under X-ray imaging, it was shown that Computer analysis of the data obtained from each CT slice and CT scans provide the accurate individualized data for CAD/CAM. In accordance to the X-Ray and CT images, it has been trying to design an optimal-fit prosthesis. The reconstruction of a three-dimensional canal model using an edge-detection system of the canal contour and CT image data for designing the prosthesis; data were analyzed and the prosthesis was designed.

The canal of the proximal femur above the lesser trochanter was mainly composed of cancellous bone sectioned obliquely on the CT scans, making the relative contrasts in this region unclear. Femoral calar existed in this region and played an important role in the force transfer and stability of the prosthesis: therefore, contours of the calar lateral canal could not be neglected in the process of designing the prostheses $[19,20]$.

The design of the prosthesis neck varies in accordance to the differences in the prosthesis head. Solidworks commercial software can design threedimensional models, which can be displayed and viewed in any plane at any level. This advanced technique enables us to modify our previous design and achieve an optimal insertable prosthesis. The design can be input into the computerized numerically controlled milling machine to produce the prosthesis.

The value of the custom prosthesis research lies in the accumulation of designed custom data and replacement cases. Through these data and cases, we can prove that CAD/CAM custom joint is both scientific and practical. In addition, we can address other problems that exist in the replacement of prosthesis. By doing so, we may modify previous designs and achieve an individualized optimal-fit design for each patient. Further research is needed in the areas of realistic prosthesis structures, prosthesis-bone contact, and the fit of prosthesis to bone. CAD/CAM is a very promising and practical way to promote the application of custom prosthesis and the development of artificial joints.

Sizes and geometries of femur bones obtained from the X-Rays and CT scans images, then calculated as data input for the development of femoral stem solid models. Figure 8 shows typical femoral stems designed with size and geometry taken from results of data provided by X-Ray and CT scan.

In this work, materials used for femoral stem for numerical simulation purposes could vary from stainless steel to Titanium, depend on the purpose of the simulation itself. For our further work, femoral stem made of Titanium Alloy will be of our concern to manufacture. Design of femoral stem with the use of Titanium Alloys is still under development. The reason of a special design for Titanium femoral stem is that on manufacturing process as well as shape and size of Titanium used

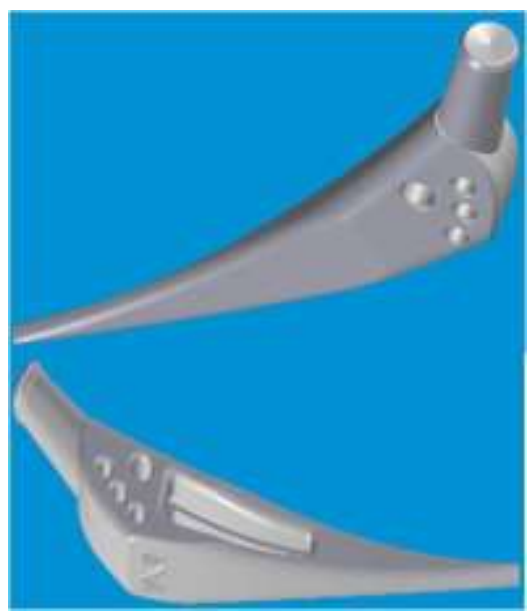

Figure. 8 Femoral stem of Total Hip Joint Arthroplasty designed in this work

Computational design of femoral stem, dedicated for Titanium alloys is shown in Figure 9. The stem consists of three separable parts, upper, lower and femoral neck parts. The design was developed in this work to get benefit from machining and materials costs, and from implantation and revision of THA processes.

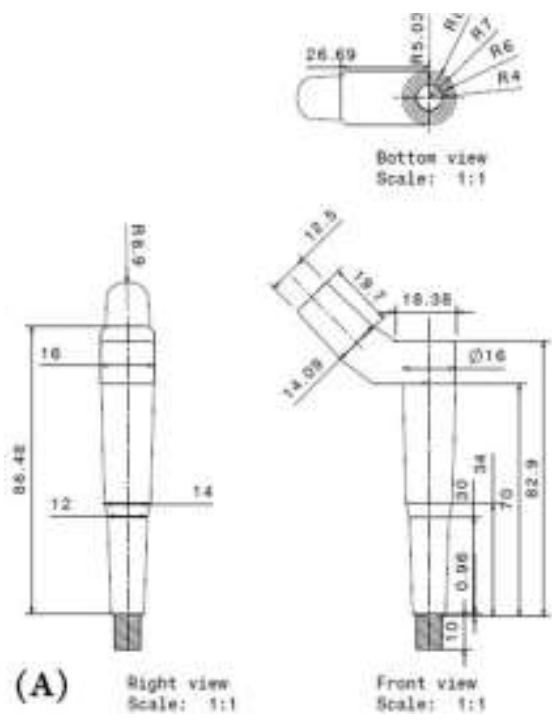

Figure .9 Modular type of upper part of femoral stem designed for Titanium Alloys

Figure 10 shows the solid model of femoral stem designed for the use of Titanium Alloy as femoral stem material. The chosen of Titanium in the form of rod shape is due to the simplicity in its manufacture process and minimizing the disposal of materials from cutting processes. Another advantage could taken by surgeons from the design developed is that with a modular type of femoral stem, it is expected to help them during revision of total hip joint Arthoplasty process. 


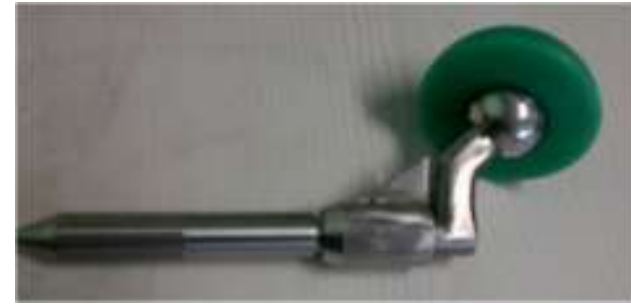

Figure.10 Femoral stem of Total Hip Joint Arthroplasty designed for Titanium Alloys

\section{CONCLUSION}

CT-scan is a powerful method to observe internal of human body, including femur bone. By using the technique, from the observation it can be seen that bone with the bright white and in great internal detail. CT is best for looking at bone fractures, and a fracture can be seen on this picture;

Reconstruction of femur bone, by observing crosssectional shapes of the canal below the trochanter gave information of the femoral bone cross section are round or elliptic. The contrast between the cortex and canal showed on CT were clear, therefore it was easy for a computer to perform the edge-detection and obtain a continuous contour.

The rendered three-dimensional canal model had a lateral "s" shaped curvature, and the upper part was wider than that of the lower part, which was shaped like a funnel. After the extraction process, a contour of the prosthesis was obtained. In the insertion process, the contour of the stem appeared similar to the canal, but the former was only optimally within the latter's contour.

Computer analysis of CT slice and CT scans could be used to provide the accurate individualized data for CAD/CAM. The X-Ray and CT images could be used to design an optimal-fit prosthesis. Finally, the reconstruction of a three-dimensional canal model can be performed by using an edge-detection system of the canal contour and CT image data for designing the prosthess.

For the use of Titanium, a special design was also introduced. This is due to for simplify the process of manufacture and for minimizing disposed materials from cutting processes; and finally for making it easier during revision process of total hip joint arthroplasty.

\section{ACKNOWLEDGEMENTS}

The author wish to thank Ministry for Research and Technology in the scheme of Incentive Programme of Applied Research for their financial support and Agency for the Assessment and Application of Technology, Centre for Materials Technology for its help during the preparation of this paper. Thanks also to Dr. Ferdiansyah and associates at Dr. Soetomo Hospital for their contribution in providing us data of hip joint anatomy in form of CT-Scan of hip joint.

\section{REFERENCES}

[1] B. R. Rawal, et al., "Design and manufacturing of femoral stems for the Indian population," Journal of Manufacturing Process, vol. 14, pp. 216 - 223, 2012.

[2] Hoaglund and W. Low, "Anatomy of the femoral neck and head with comparative data from Caucasian and Kong Cheenese " Clinical Orthopaedics and Related Research, vol. 10, p. 152, 2005.

[3] A. M. Riesgo, et al., "Survivorship and Complications of Revision Total Hip Arthroplasty with a Mid-Modular Femoral Stem," The Journal of Arthroplasty, 2015.

[4] Gotze, et al., "CT-based accuracy of implanting custom-made endoprostheses," Clinical Biomechanics, vol. 20, pp. 856 - 862 , 2005

[5] Q. Lian, et al., "Patient-Specific Design and Biomechanical Evaluation of a Novel Bipolar Femoral Hemi-Knee Prosthesi," Journal of Bionic Engineering, vol. 11, pp. 259 - 267, 2014.

[6] D. Robertson, et al., "Improving the fit of press-fit hip stems," Clinical Orthopaedics and Related Research, vol. 228, pp. 134 140, 1988.

[7] S. Stulberg, et al., "Design characteristics and preliminary results of primary custom total hip prosthesis " Clinical Orthopaedics and Related Research, vol. 249, pp. 79 - 96, 1989.

[8] R. M. Gillies, et al., "The influence of design parameters on cortical strain distribution of a cementless titanium femoral stem," Medical Engineering \& Physics, vol. 24, pp. 109 - 114, 2002.

[9] H. Gong, et al., " An adaptation model for trabecular bone at different mechanical levels.," Biomedical Engineering Online, vol. 9 , p. 32, 2010.

[10] M. O. Heller, et al., "Musculo-skeletal loading conditions at the hip during walking and stair climbing," Journal of Biomechanics, vol 34 , pp. 883 - 932001.

[11] K. B. Hazlehurst, et al., "An investigation into the flexural characteristics of functionally graded cobalt chrome femoral stems manufactured using selective laser melting," Materials \& Design, vol. 60 , pp. 177 - 183, 2014

[12] G. Yamako, et al., "Load-transfer analysis after insertion of cementless anatomical femoral stem using pre- and post-operative CT images based patient-specific finite element analysi," Medical Engineering \& Physics, vol. 36, pp. 694 - 700, 2014.

[13] B. T. Barlow, et al., "Short-Term Metal Ion Trends Following Removal of Recalled Modular Neck Femoral Stems," The Journal of Arthroplasty, vol. 30, pp. 1191 - 1196, 2015.

[14] B. T. Palumbo, et al., "Results of Revision Total Hip Arthroplasty with Modular, Titanium-Tapered Femoral Stems in Severe Proximal Metaphyseal and Diaphyseal Bone Loss," The Journal of Arthroplasty, vol. 28, pp. 690 - 694, 2013.

[15] I. Chow, et al., "Short Stem Metaphyseal-Engaging Femoral Implants: A Case-Controlled Radiographic and Clinical Evaluation with Eight Year Follow-Up," The Journal of Arthroplasty, vol. 30, pp. 600 - 606, 2015.

[16] M. Biegler, et al., "Effect of porous coating and loading condi-tions on total hip femoral stem stability," J Arthroplasty, vol. 10, pp. 839 - 847, 1995.

[17] H. J. Laine, et al., "Diversity of proximal femoral modullary canal," Journal of Arthroplasty, vol. 15, pp. 86 -92 2000.

[18] L. SportsOrthopaedics. (2015, September 5th, 2015). Hip Arthroscopy. Available: www.sportortho.co.uk/mobile/leftnavigation

[19] Dai KR, et al., "Geometric and biomechanical analysis of the human femur," Orthop Trans, vol. 10, p. 99, 1985.

[20] M. Brncick, "Computer automated design and computer automated manufacture," Phys Med Rehabil Clin N Am, vol. 11, pp. 701-713, 2000 